\title{
Correction: Survivin Mutant Protects Differentiated Dopaminergic SK-N-SH Cells Against Oxidative Stress
}

\section{Sara Baratchi, Rupinder K. Kanwar, Jagat R. Kanwar}

The authors would like to correct Fig 3. In Fig 3, the panels used for 3A-C are incorrectly taken from the same micrograph as 3G-I. The authors have provided a corrected version of Fig 3 here. The authors confirm that these changes do not alter their findings.

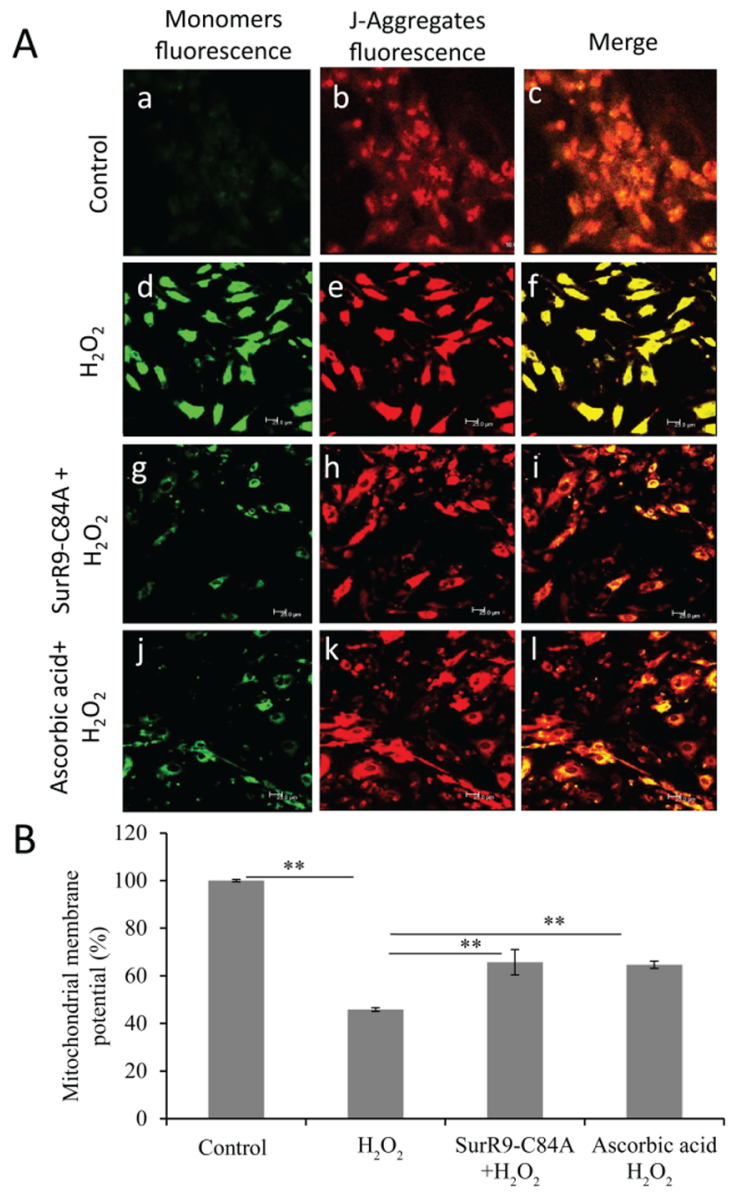

Fig 3. SurR9-C84A prevents mitochondrial depolarization. SK-N-SH cells were differentiated with $20 \mu \mathrm{M}$ retinoic acid for 10 days. Differentiated media were replaced with growth media and cells were pre-treated with $75 \mu \mathrm{g} / \mathrm{ml}$ of SurR9-C84A or ascorbic acid for $24 \mathrm{hr}$ followed by treatment with $300 \mu \mathrm{M}$ of $\mathrm{H}_{2} \mathrm{O}_{2}$ for $24 \mathrm{hr}$. At the end of incubation mitochondrial membrane depolarization was qualified and quantified with MitoLight Mitochondrial kit using both techniques of (A) confocal microscopy and (B) spectrofluorometery (see material and method). Green fluorescence (detection of monomers) indicates the presence of depolarized mitochondria (apoptotic cells). Red fluorescence (Jaggregates) indicates the functional and polarized mitochondria. Values are presented as a percentage of increase in mitochondrial depolarization. Data are representative of at least three independent experiments and expressed as mean $\pm \mathrm{SEM} ;{ }^{*} \mathrm{P}<0.05,{ }^{* *} \mathrm{P}<0.01$.

https://doi.org/10.1371/journal.pone.0194587.g001 


\section{Supporting information}

S1 File. Image used in panels 3G-I.

(JPG)

S2 File. Correct image for panels 3A-C.

(JPG)

\section{Reference}

1. Baratchi S, Kanwar RK, Kanwar JR (2011) Survivin Mutant Protects Differentiated Dopaminergic SK-N-SH Cells Against Oxidative Stress. PLoS ONE 6(1): e15865. https://doi.org/10.1371/journal. pone.0015865 PMID: 21249229 